
Copyright $\odot 2021$ World Federation for Ultrasound in Medicine \& Biology. All rights reserved. Printed in the USA. All rights reserved. $0301-5629 / \$$ - see front matter

https://doi.org/10.1016/j.ultrasmedbio.2021.03.018

\title{
AUTOMATED SEGMENTATION OF THE MEDIAN NERVE IN THE CARPAL TUNNEL USING U-NET
}

\author{
Raymond T. Festen, ${ }^{*}$ Verena J.M.M. Schrier, ${ }^{*}, \dagger$ and Peter C. Amadio* \\ * Department of Orthopedic Surgery, Mayo Clinic, Rochester, Minnesota, USA; and ${ }^{\dagger}$ Department of Plastic, Reconstructive and \\ Hand Surgery, Erasmus Medical Center, Rotterdam, The Netherlands
}

\begin{abstract}
Nerve area and motion in carpal tunnel syndrome (CTS) are currently under investigation in terms of prognostic potential. Therefore, there is increasing interest in non-invasive measurement of the nerve using ultrasound. Manual segmentation is time consuming and subject to inter-rater variation, providing an opportunity for automation. Dynamic ultrasound images $(n=5560)$ of carpal tunnels from 99 clinically diagnosed CTS patients were used to train a U-Net-shaped neural network. The best results from the U-Net were achieved with a location primer as initial region of interest for the segmentations during finger flexion (Dice coefficient $=0.88$ ). This is comparable to the manual Dice measure of 0.92 and higher than the resulting automated Dice measure of wrist flexion (0.81). Although there is a dependency on image quality, a trained U-Net can reliably be used in the assessment of ultrasound-acquired median nerve size and mobility, considerably decreasing manual effort. (E-mail: pamadio@mayo.edu) (c) 2021 World Federation for Ultrasound in Medicine \& Biology. All rights reserved.
\end{abstract}

Key Words: Ultrasound, U-Net, Carpal tunnel, Median nerve, Segmentation.

\section{INTRODUCTION}

Carpal tunnel syndrome (CTS) is characterized by tingling and numbness in the distribution area of the median nerve caused by compression at the carpal tunnel. No widely accepted gold standard for CTS diagnosis exists. Commonly, CTS diagnoses are made based on the combination of clinical symptoms, physical examination and neuroconductive studies (NCSs) with or without electromyography (American Academy of Orthopedic Surgeons [AAoS] 2016). On the basis of severity, local guidelines and patient preference, treatment can include splinting, corticosteroid injections and/ or surgical division of the transverse carpal ligament.

Ultrasonography has emerged as a relevant imaging modality for patients with CTS. Currently, it is used mostly as an addendum to the classic NCS during the diagnostic workup, with sensitivity and specificity reaching similar levels (Fowler et al. 2011) and high (intra-class correlation coefficient $[\mathrm{ICC}]>0.9)$ intra-rater reliability (Ghasemi-Esfe et al. 2011; El Miedany et al. 2015; Fowler et al. 2015; Gonzalez-Suarez et al. 2018). Studies indicate, however, that inter-rater reliability is more prone to variation, with reported ICC values between 0.57 and

Address correspondence to: Peter C. Amadio, Tendon and Soft Tissue Biology Laboratory, Mayo Clinic, 200 1st Street SW, Rochester, MN 55905, USA. E-mail: pamadio@mayo.edu
0.94 (Van Doesburg et al. 2010; GonzalezSuarez et al. 2018; Schrier et al. 2019). Although NCSs serve as a direct measure for alterations in the function of the nerve, it is a test with limited ability to predict clinical severity or intervention outcome (Finsen and Russwurm 2001; Naranjo et al. 2009; AAoS 2016). Ultrasound allows direct visualization of the nerve, its position in the carpal tunnel and its relation to the surrounding structures, making it a helpful guidance tool, for example, during injection (Evers et al. 2017). Research has also suggested that median nerve cross-sectional area (CSA) at the level of the carpal tunnel inlet is a reliable diagnostic feature (Buchberger et al. 1991; Wong et al. 2004; Yesildag et al. 2004; Ziswiler et al. 2005). In addition to size, there is a decrease in nerve mobility in CTS patients (Nakamichi and Tachibana 1995; Erel et al. 2003; Van Doesburg et al. 2012; Wang et al. 2014; Nanno et al. 2015; Kuo et al. 2016; Ellis et al. 2017; Schrier et al. 2019), which can be quantified using dynamic ultrasound.

Segmentation of the nerve not only helps to acquire nerve size, but also allows for tracking during finger and wrist motion. Patients with CTS often have edematous, fibrotic connective tissue surrounding the median nerve, causing inhibition of nerve motion. Nanno et al. (2017) reported significant changes in median nerve motion after CTR. Nerve dynamics 
may thus hold useful information pertaining to CTS pathophysiology, but so far there has been limited research associating this with clinical outcome. Segmentation allows for frame-to-frame tracking of the nerve, visualizing nerve pathways and allowing for quantification of nerve motion.

With the increase in interest in using US in different facets of CTS patient assessment, an automated nerve segmentation method could aid in the reliability of the measure, decrease time and cognitive efforts and decrease inter-rater variability.

\section{METHODS}

\section{General}

Data used in this study are derived from a registered prospective cohort study (Clinicaltrials.gov: NCT02219555) which was reviewed and approved by the institutional review board (IRB No. 14-003444). This study was aimed at assessing the prognostic value of dynamic ultrasound by measuring the median nerve mobility and connective tissue response in CTS patients before treatment. Written informed consent was obtained from all participants in accordance with the Declaration of Helsinki (World Medical Association 2013).

\section{Data set and ultrasound protocol}

Images were collected at baseline and 1 or 3 months after corticosteroid injection or surgical intervention, respectively. Idiopathic CTS patients were evaluated by specialized physicians at a single tertiary medical center with a dedicated hand clinic. Patients were recruited if they met the following inclusion criteria: clinical diagnosis of CTS (based on classic symptoms including numbness or tingling in the distribution area of the median nerve), age between 21 and $80 \mathrm{y}$, symptom duration for at least $4 \mathrm{wk}$ in at least two digits on one hand and a full understanding of English language. Exclusion criteria were a previous surgical release, tumor, mass or deformity in the hand/wrist, pregnancy-related CTS and any of the following diagnoses: cervical radiculopathy, peripheral nerve disease, thyroid disease, rheumatoid arthritis or other inflammatory arthritis, osteoarthritis in wrist, diabetes, renal failure, sarcoidosis, amyloidosis or major trauma to ipsilateral hand or wrist. In patients who experienced bilateral CTS, only the hand with the most severe (self-reported) symptoms was included.

Images were taken just proximal to the level of the carpal tunnel inlet defined as the proximal margin of the flexor retinaculum (Fig. 1a). The nerve was identified by its shape (oval) with a hyperechoic delineation (epineurium) and a "honeycomb"-like appearance caused by the perineurial septations between the fascicles. Patients were asked to flex their fingers and their wrists in two separate motions. Generally, the median nerve will move in the dorso-ulnar direction (Fig. 1b). All images were made using a Philips iE33 (Royal Philips Electronics, Amsterdam, Netherlands) ultrasound machine with a L15-7io linear transducer. Three sonographers were involved in acquisition of the images. Two sonographers were residents with $1 \mathrm{y}$ of ultrasound experience. They were trained for multiple weeks by a dedicated musculoskeletal sonographer (10+ years of experience), all blinded to any clinical outcome measures at the time of acquisition. Measurements were performed according to a strict protocol designed specifically for this study by the musculoskeletal sonographer. Manual analysis of the DICOM images was done using in-house software (Analyze Version 12.0, Biomedical Imaging Resource, Mayo Clinic, Rochester MN, USA) by two experienced researchers who demarcated the median nerve using a polygon. A gross representation of nerve mobility was needed, so segmentation was performed 11 times per cine loop using 33 frame intervals. From 99 patients with an average of 5.1 videos, a library was created containing 505 videos with 5560 annotated frames. For the
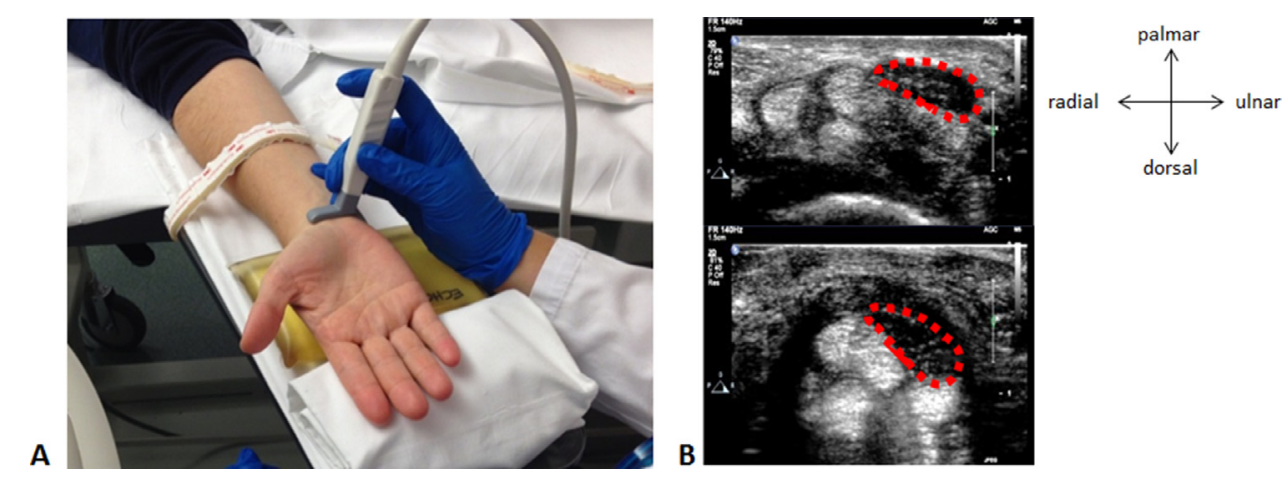

Fig. 1. (a) The probe was positioned just proximal to the carpal tunnel inlet, with the patient's hand strapped to a board while supported with a gel pad and kept in neutral position using towels. (b) Example ultrasound image of median nerve before (upper) and after wrist motion (lower). Note the typical dorso-ulnar translation. 
training of U-Net, images were sampled from 3336 (60\% of the total) frames. One thousand one hundred twelve $(20 \%)$ images were used for validation, and $1112(20 \%)$ for tests.

\section{Neural Network design and software}

The ultrasound videos were converted from DICOM to PNG format using MATLAB 9.5 (The MathWorks, Natick, MA, USA). Subsequent parts of the system were programmed in Python 3 (Open source, Python Software Foundation, Beaverton, OR, USA) using a.o. Numpy and the TensorFlow 1.5 (Open source, Google, Mountain View, CA, USA) libraries.

An Artificial Neural Network, a U-Net model, was adapted from Ronneberger et al. (2015). Two implementations of the U-Net model were trained: The first model is based on single-frame segmentation. In this model, every single frame of a video is assessed individually without prospective information or feedback from earlier segmented frames. A second version of the model was made using focus windows. In this model, spatial information from the previous segmented frame is used to redirect the focus of the search area for the next frame, hypothetically increasing the odds of a correct segmentation. The first step in this model was to crop the frame to the relevant section - focus window-of the image.

Tests were performed with the segmentation of the first frame done manually and with the first frame segmented by the single-frame network (referred to as the third model, "fully automated").

Figure 2 illustrates the overall layout of the U-Net model starting with a single image entering at the top left. A single frame (.png file) was downsized from $1024 \times 768$ to $512 \times 384$ pixels (in the single-frame model) or, as in Figure 2, cropped to $320 \times 240$ pixels around the nerve when using focus windows. On the downslope - left side — of the U-shaped model, the information of the image is transformed with an added dimension (z-axis) containing abstract information and the condensed shape in the $x$ - and $y$-directions, becoming smaller in the $\mathrm{x}$ - and $\mathrm{y}$-directions and deeper in the $\mathrm{z}$ direction. On the upslope - right side-of the U-shape the information is scaled back up to the original size of the image with a single depth, where the segmentation emerges with help of the location information from the same-sized shape from the left side as depicted by the horizontal arrows across the ' $U$ '. Finally, the image with the segmentation of $320 \times 240$ is positioned back in the $1024 \times 768$ image where it was cropped from. The arrows depicting convolutional filters and transpose convolutional filters are convolution operations with parameters that are trained using the neural network methodology, making UNet a convolutional neural network.

The U-Net model used for the initial frame of the video uses images and masks of $512 \times 384$, the U-Net model used for the consecutive frames uses images and masks of $320 \times 240$ (no compression) unless the size of a nerve is larger than the $320 \times 240 * 0.25 \mathrm{~mm} /$ pixel $=8$ $\mathrm{mm} \times 6 \mathrm{~mm}$.

The model with the focus window uses the mask of frame $n-1$ to determine the focus area for frame $n$. For the training set, however, the 11 annotated frames from the total of 334 frames in the video were not consecutive frames but 33 frames apart. To train the model to be adaptable to a moving nerve, as is seen during finger and wrist flexion, the focus window was subjected to a random geometric translation around the known mask $n$ (Fig. 3).

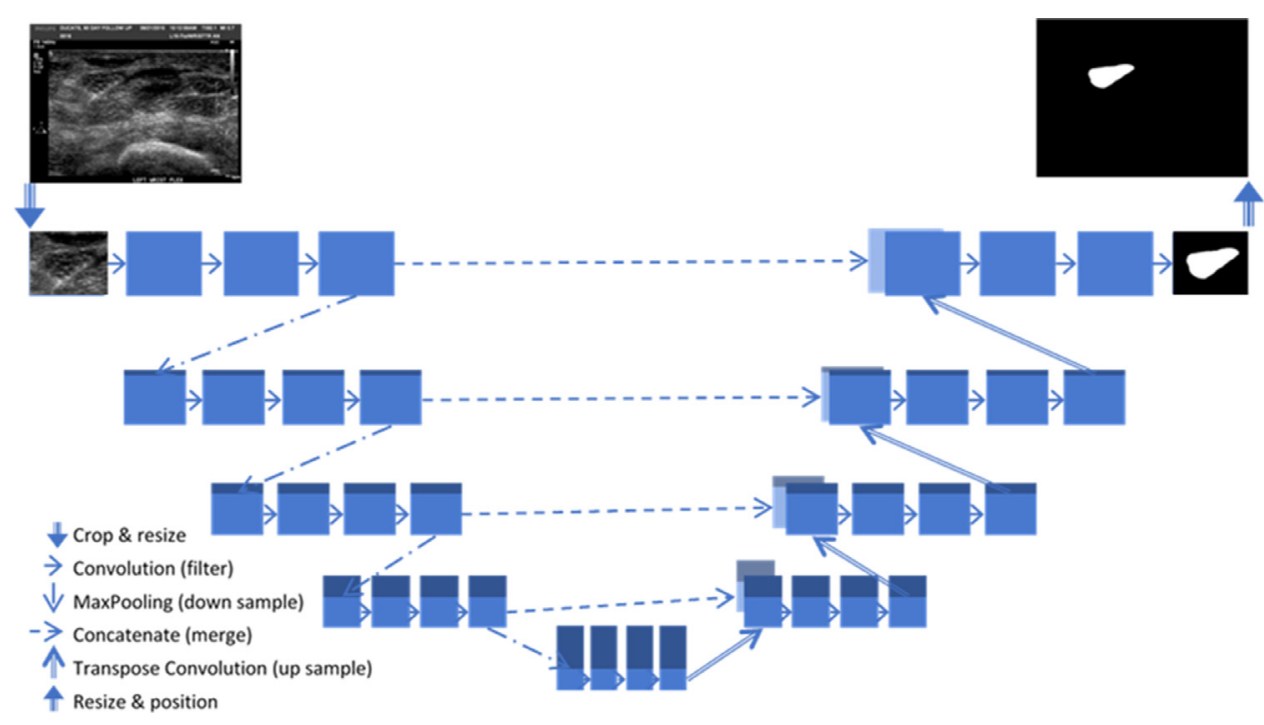

Fig. 2. Diagram of U-Net model. 

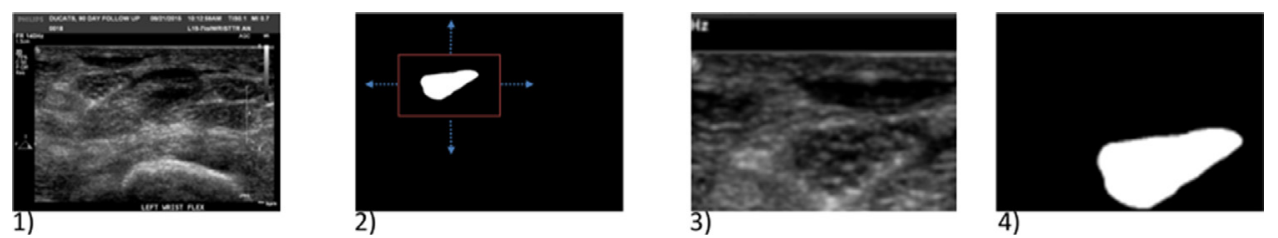

Fig. 3. From left to right: (1) Original frame $n$. (2) Truth mask $n$ with a focus window to be translated randomly. (3) Translated focus window cut from frame $n$. (4) Cut from mask $n$.

\section{Primary outcome}

During the training, as well as the final comparison with the manual segmentation, the mean Dice coefficient (95\% confidence interval) was used as primary outcome. This measure represents the relative overlap of two segmentations, with " 0 " indicating no overlap at all and " 1 " representing perfect overlap. This measure therefore represents both morphological and spatial accuracy.

\section{RESULTS}

The resulting Dice coefficients for both models are listed in Table 1 . The first model was marginally outperformed by the focused frame model, and only if the first frame was defined manually by a user ( 0.82 vs. 0.85$)$. Additionally, better results were acquired using images gained during finger flexion irrespective of U-Net model, but most prominently seen in the focused frames - manual approach (0.88 vs. 0.81). During the design it was noted that in particular cases, especially where the nerve exhibited larger excursions and the quality of the images was diminished, the resulting masks were less accurate, leading to a cascade of failed segmentations. After automated segmentation, the system produced an assortment of data for each frame of the video, providing potential research metrics (Fig. 4).

By use of the center point of the manual/automated overlay of the nerve, nerve size, perimeter and absolute coordinates were abstracted. The coordinates were used to track the nerve and calculate the overall motion (see Supplementary Video A [online only] for an example).

\section{DISCUSSION}

This study found that a U-Net model can be used to accurately assess 2-D median nerve characteristics and mobility in ultrasound-based images using a medium- sized data set. Performance can be enhanced by using a single manual segmentation to add prospective spatial information to the model.

In clinical practice, the system can aid in getting to a consistent method of diagnosing CTS using ultrasound. Another purpose of the model is to aid in predicting outcomes of CTS remedies. The computer easily outputs various data concerning the nerve: next to the standard measures area and perimeter, the movement of the center of the nerve compared with the previous frame or the change in shape compared with previous frame. With the model, this can be done for all the images in a video clip, creating a source of information on the dynamics of the nerve that, together with other structured data such as patient specifics and possibly existing NCS data, can be used to dial in on a predictive model informing both physician and patient on chances of success, facilitating their joint decision-making process.

With decreased nerve mobility as a potential marker for CTS diagnosis (Ellis et al. 2017) as well as prognosis in those undergoing injection (Schrier et al. 2019), the results of our study help in the analysis and assessment of the true added value of nerve mobility in clinical evaluation. Studies indicate that intra-rater reliability generally is high (ICC >0.9) when measuring the nerve crosssectional area, but inter-rater reliability is lower, with reported ICC values between 0.57 and 0.94 (Van Doesburg et al. 2010; Gonzalez-Suarez et al. 2018; Schrier et al. 2019). Because the intra-rater performance of a U-Net model is 1.00 , its added value is limited only by its accuracy. Through comparison of the accuracy with the aforementioned inter-rater accuracies starting at 0.57 , the system performed better with scores up to 0.88 . Still the main advantages are the increased number of measurements, consistency of measurement, as the algorithm will always provide the same segmentation, and

Table 1. Dice coefficients ( $95 \%$ confidence interval) for separate U-Net strategies

\begin{tabular}{llll}
\hline \multirow{2}{*}{ Data set } & (1) Single frames & \multicolumn{2}{c}{ Focus windows } \\
\cline { 3 - 4 } & & (2) Manual first frame & (3) First frame defined by "single frames" U-Net \\
\hline Finger flexion $(\mathrm{n}=594)$ & $0.86(0.85-0.88)$ & $0.88(0.87-0.89)$ & $0.83(0.81-0.86)$ \\
Wrist flexion $(\mathrm{n}=518)$ & $0.77(0.75-0.80)$ & $0.81(0.79-0.83)$ & $0.80(0.78-0.82)$ \\
Both $(\mathrm{n}=1112)$ & $0.82(0.81-0.84)$ & $0.85(0.84-0.86)$ & $0.82(0.81-0.83)$ \\
\hline
\end{tabular}



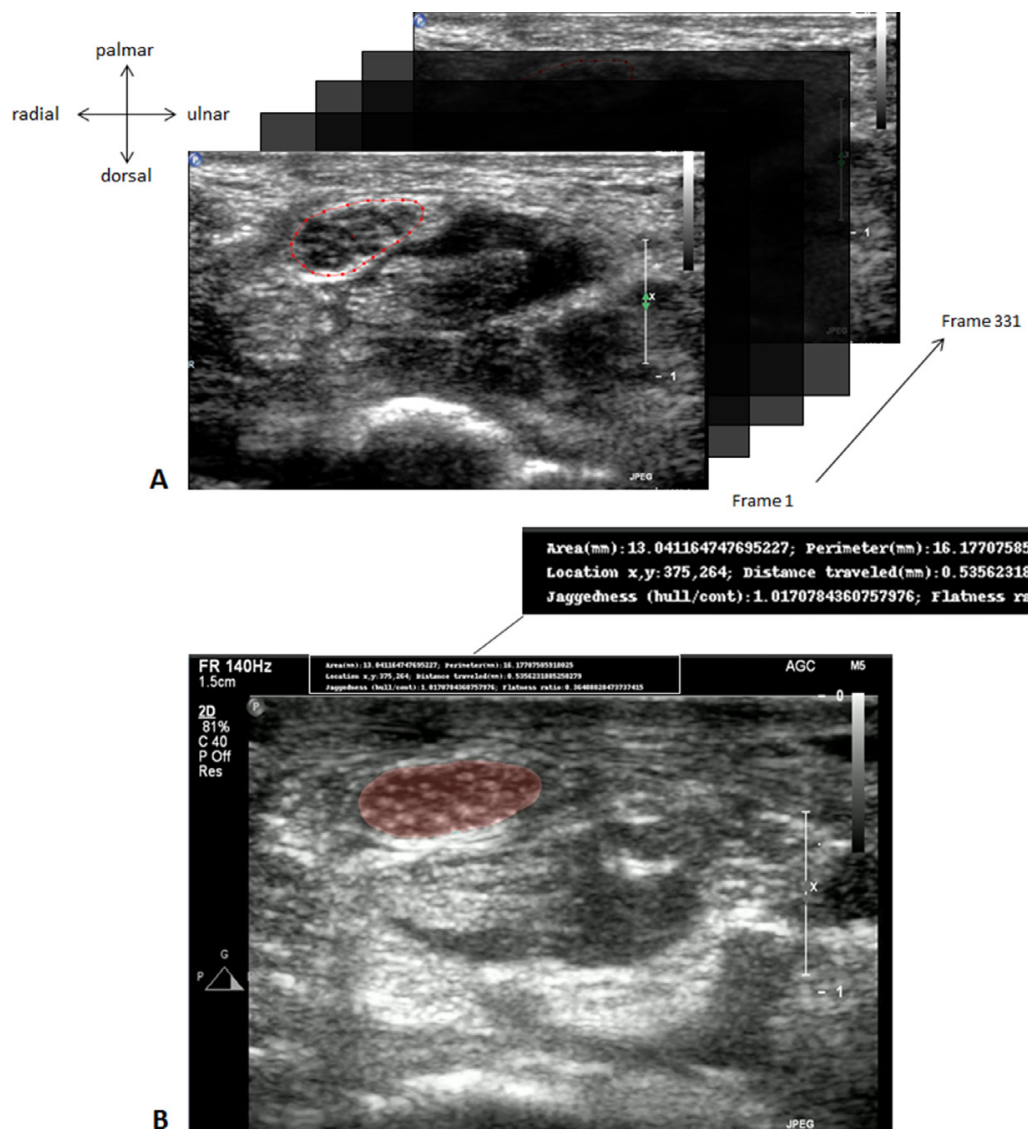

Fig. 4. (a) Example of an ultrasound image manually segmented (red dotted outline). A cine clip of 331 frames was recorded and manually segmented every 32 nd frame to acquire 11 data points to quantify the nerve's motion. (b) Example of an ultrasound frame with an automated overlay in orange. At the top is a real-time display of several nerve parameters including area, perimeter and location coordinates.

speed. With an optimized hardware setup the analysis should be finished within minutes and be well applicable in the context of patient examination.

A challenge in dynamic measurements with US is the change in image features during motion created by the normal anisotropic characteristics of the nerve. Moreover, the shape of the nerve resembles the flexor tendons present in the carpal tunnel. Where a human interpreter would use anatomical knowledge and positive feedback tests (patient moving a finger), our model does not have this information. Consequently, the system can err from the truth. For practical usability it is important to know when this happens so the outlier can be eliminated from further analysis or perhaps a new clip of higher quality can be recorded. A way to cope with this is to add a visual check of the resulting video clip of $7 \mathrm{~s}$. Improvements to the algorithm can be made, for instance, by employing Active Contour Model as postprocessing step. If the visibility of the nerve can be maintained with wrist flexions, the model will perform better for those as well. Preprocessing of images for sharpening, denoising, and so forth, is done by convolutional filters. As U-Net is a convolutional network, ideally its contents already cover those functionalities. However, established filters from classic - non-artificial intelligence - computer image analysis as a preprocessing step before U-Net could be tested to improve the results.

Limitations of this study are the single ultrasound system that was used; other probes will yield different images and will need additional training for the network to perform. Chances are there that the model misses the targeted segmentation. A visual check by the physician in the clinical context and an outlier detector in research context are recommended.

For future research we can look at the model itself with added preprocessing and postprocessing to increase accuracy. To extend the use of the model as a practical tool for a physician, it can be further developed to create a 3-D image of the median nerve. Finally, to use the information from the model to predict the probability of treatments being successful, further research is needed to 
link the attained data with, for instance, NCSs of historic outcomes of those treatments.

Acknowledgments-The authors acknowledge the U.S. National Institutes of Health $(\mathrm{NIH})$ for providing funding for this work. The NIH was not involved in the design of the study, collection of data, manuscript writing or decision to publish.

Conflict of interest-None of the authors has a financial conflict related to the content of this work.

\section{SUPPLEMENTARY MATERIALS}

Supplementary material associated with this article can be found in the online version at doi:10.1016/j.ultra smedbio.2021.03.018.

\section{REFERENCES}

American Academy of Orthopedic Surgeons (AAOS). Management of carpal tunnel syndrome: Evidence-based clinical practice guideline. February 29, 2016. Available at: https://aaos.org/globalassets/qual ity-and-practice-resources/carpal-tunnel/management-of-carpal-tun nel-syndrome-7-31-19.pdf

Buchberger W, Schön G, Strasser K, Jungwirth W. High-resolution ultrasonography of the carpal tunnel. J Ultrasound Med 1991;10:531-537.

El Miedany Y, El Gaafary M, Youssef S, Ahmed I, Nasr A. Ultrasound assessment of the median nerve: A biomarker that can help in setting a treat to target approach tailored for carpal tunnel syndrome patients. Springerplus 2015;4:13.

Ellis R, Blyth R, Arnold N, Miner-Williams W. Is there a relationship between impaired median nerve excursion and carpal tunnel syndrome? A systematic review. J Hand Ther 2017;30:3-12.

Erel E, Dilley A, Greening J, Morris V, Cohen B, Lynn B. Longitudinal sliding of the median nerve in patients with carpal tunnel syndrome. J Hand Surg 2003;28:439-443.

Evers S, Bryan AJ, Sanders TL, Selles RW, Gelfman R, Amadio PC. Effectiveness of ultrasound-guided compared to blind steroid injections in the treatment of carpal tunnel syndrome. Arthritis Care Res 2017;69:1060-1065.

Finsen V, Russwurm H. Neurophysiology not required before surgery for typical carpal tunnel syndrome. J Hand Surg 2001;26:61-64.

Fowler JR, Gaughan JP, Ilyas AM. The sensitivity and specificity of ultrasound for the diagnosis of carpal tunnel syndrome: A metaanalysis. Clin Orthop Relat Res 2011;469:1089-1094.

Fowler JR, Hirsch D, Kruse K. The reliability of ultrasound measurements of the median nerve at the carpal tunnel inlet. J Hand Surg 2015;40:1992-1995.

Ghasemi-Esfe AR, Khalilzadeh O, Vaziri-Bozorg SM, Jajroudi M, Shakiba M, Mazloumi M, Rahmani M. Color and power Doppler US for diagnosing carpal tunnel syndrome and determining its severity: A quantitative image processing method. Radiology 2011;261:499-506.

Gonzalez-Suarez CB, Buenavente MLD, Cua RCA, Fidel MBC, Cabrera JTC, Regala CFG. Inter-rater and intra-rater reliability of sonographic median nerve and wrist measurements. J Med Ultrasound 2018;26:14.

Kuo TT, Lee MR, Liao YY, Chen JP, Hsu YW, Yeh CK. Assessment of median nerve mobility by ultrasound dynamic imaging for diagnosing carpal tunnel syndrome. PloS One 2016;11 e0147051.

Nakamichi K, Tachibana S. Restricted motion of the median nerve in carpal tunnel syndrome. J Hand Surg 1995;20:460-464.

Nanno M, Sawaizumi T, Kodera N, Tomori Y, Takai S. Transverse movement of the median nerve in the carpal tunnel during wrist and finger motion in patients with carpal tunnel syndrome. Tohoku J Exp Med 2015;236:233-240.

Nanno M, Kodera N, Tomori Y, Hagiwara Y, Takai S. Median nerve movement in the carpal tunnel before and after carpal tunnel release using transverse ultrasound. J Orthop Surg (Hong Kong) 2017;25 2309499017730422.

Naranjo A, Ojeda S, Araña V, Baeta P, Fernández-Palacios Martínez J, García-Duque O, Rodríguez-Lozano C, Carmona L. Usefulness of clinical findings, nerve conduction studies and ultrasonography to predict response to surgical release in idiopathic carpal tunnel syndrome. Clin Exp Rheumatol 2009;27:786-793.

Ronneberger O, Fischer P, Brox T. U-Net: Convolutional networks for biomedical image segmentation. In: Navab $\mathrm{N}$, Hornegger J, Wells W, Frangi A, (eds). Medical Image Computing and Computer-Assisted Intervention: MICCAI 2015. Lecture Notes in Computer Science, Vol. 9351. Cham: Springer; 2015. p. 234-241.

Schrier VJ, Evers S, Geske JR, Kremers WK, Villarraga HR, Kakar S, Selles RW, Hovius SE, Gelfman R, Amadio PC. Median nerve transverse mobility and outcome after carpal tunnel release. Ultrasound Med Biol 2019;45:2887-2897.

Van Doesburg MH, Yoshii Y, Villarraga HR, Henderson J, Cha SS, An KN, Amadio PC. Median nerve deformation and displacement in the carpal tunnel during index finger and thumb motion. J Orthop Res 2010;28:1387-1390.

Van Doesburg MH, Henderson J, Yoshii Y, Van Der Molen ABM, Cha SS, An KN, Amadio PC. Median nerve deformation in differential finger motions: Ultrasonographic comparison of carpal tunnel syndrome patients and healthy controls. J Orthop Res 2012;30:643648 .

Wang Y, Filius A, Zhao C, Passe SM, Thoreson AR, An KN, Amadio PC. Altered median nerve deformation and transverse displacement during wrist movement in patients with carpal tunnel syndrome. Acad Radiol 2014;21:472-480.

Wong SM, Griffith JF, Hui AC, Lo SK, Fu M, Wong KS. Carpal tunnel syndrome: Diagnostic usefulness of sonography. Radiology 2004;232:93-99.

World Medical Association. World Medical Association Declaration of Helsinki: Ethical principles for medical research involving human subjects. JAMA 2013;310:2191.

Yesildag A, Kutluhan S, Sengul N, Koyuncuoglu H, Oyar O, Guler K, Gulsoy U. The role of ultrasonographic measurements of the median nerve in the diagnosis of carpal tunnel syndrome. Clin Radiol 2004;59:910-915.

Ziswiler HR, Reichenbach S, Vögelin E, Bachmann LM, Villiger PM, Jüni P. Diagnostic value of sonography in patients with suspected carpal tunnel syndrome: A prospective study. Arthritis Rheum 2005;52:304-311. 